\title{
Characterization of fiber-based slit homogenizer devices in the NIR and SWIR
}

Simon Amann ${ }^{\mathrm{a}}$, Quynh Duong-Ederer ${ }^{\mathrm{a}}$, Tobias Haist $^{\mathrm{a}}$, Bernd Sierk ${ }^{\mathrm{b}}$, Benedikt Guldimann ${ }^{\mathrm{b}}$, Wolfgang Osten ${ }^{\mathrm{a}}$

aInstitut für Technische Optik, Universität Stuttgart, Pfaffenwaldring 9, 70569 Stuttgart, Germany

${ }^{b}$ ESA-ESTEC, Keplerlaan 1, Noordwijk, The Netherlands

\section{Motivation}

Satellite-based spectrometer are used for spatial monitoring of anthropogenic greenouse gases. Subpixel inhomogenities of the light intensity in the entrance slit reduces the measurement accuracy. An approach for the characterization of various fiber based homogenizer devices for reducing this problem as well as results are shown in this contribution.

\section{Light homogenization efficiency}

The fiber input is illuminated with different inhomogeneous scenes. The efficiency is then determined by the homogeneity of the near field light intensity at the fiber output. Temporal coherent (high resolution of the spectrometer) and spatial incoherent light is realilzed using a laser and a rotating diffusor. Measurements with $770 \mathrm{~nm}$ and $1620 \mathrm{~nm}$ and different polarisations have been carried out. The investigated devices are manufactured by CeramOptec. The measurement setup consists of three parts: illumination system, imaging of the scene/mask onto the homogenizer input and imaging of the fiber output onto the main image sensor.

Fiber input illumination

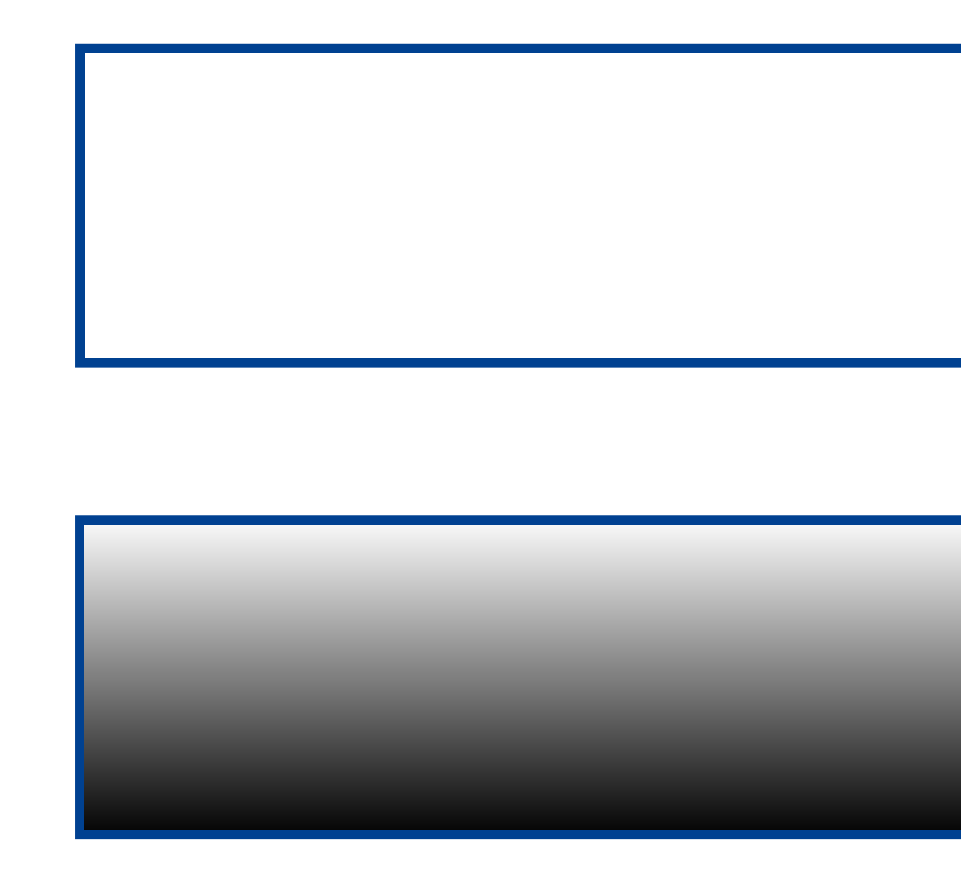

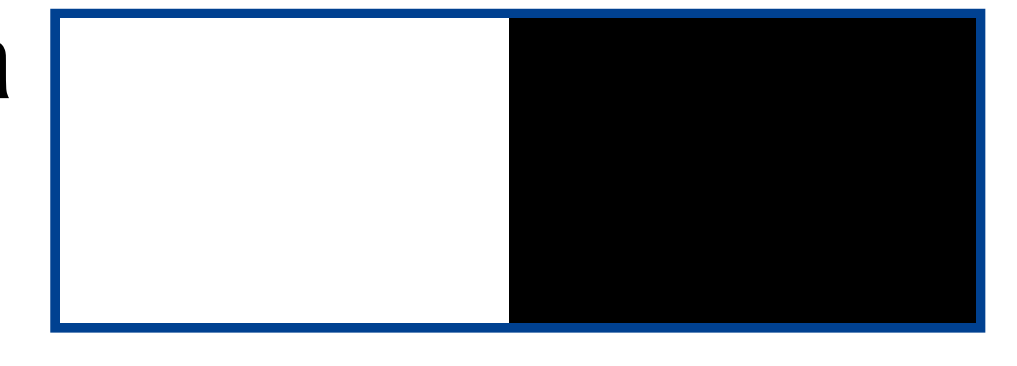

Reflectivity change across flight direction

Mixture
Reflectivity change along flight direction

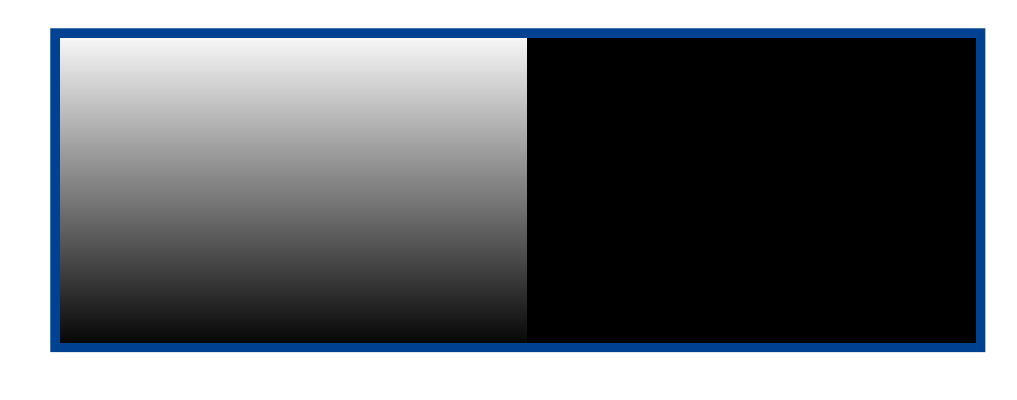

Reduction of sensor caused interference pattern by controlled movement of the sensor with an automated micro positioning stage in combination with image processing.
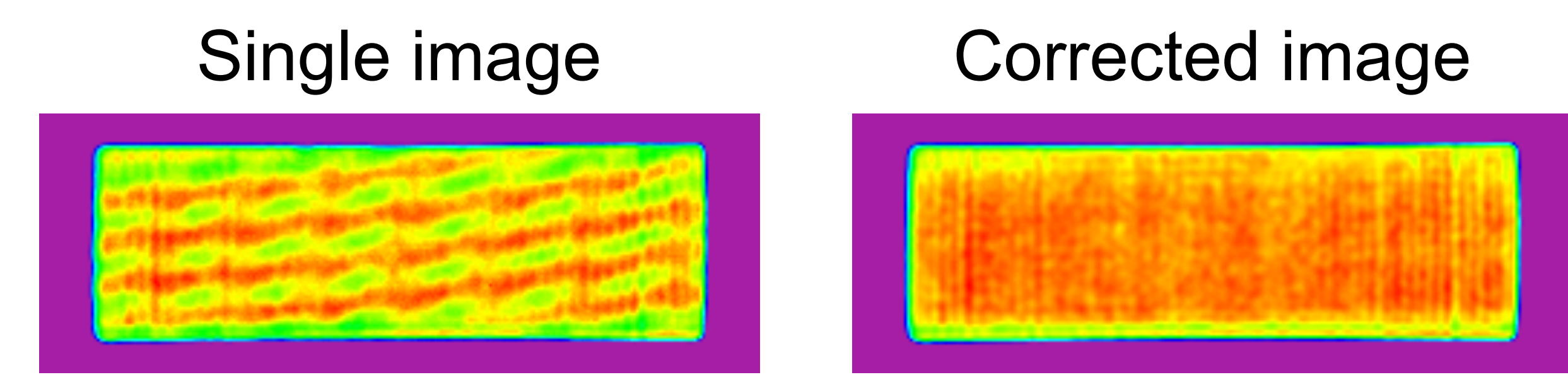

Setup

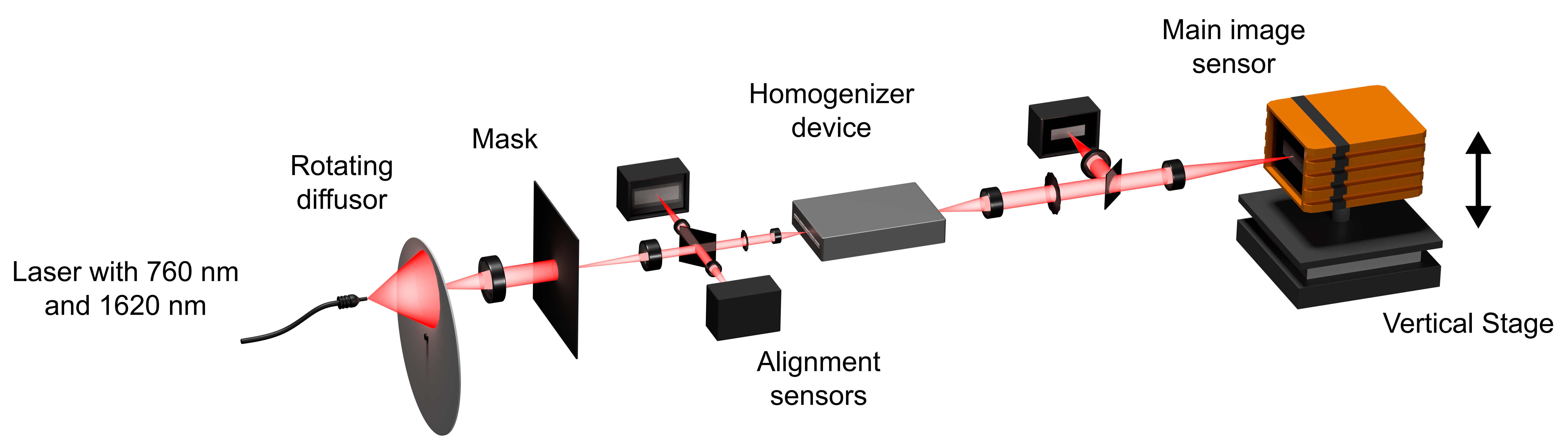

Fiber output intensity of various $100 \times 100 \mu \mathrm{m}$ and $300 \times 100 \mu \mathrm{m}$ devices.

Depolarization measurements with a homogeneous input scene. The examples show results of the $100 \times 300 \mu \mathrm{m}$ single fibers with different Single fiber, $10 \mathrm{~cm}$ and $1 \mathrm{~m}$ long Fiber array, $2 \mathrm{~cm}$ and $4 \mathrm{~cm}$ long

NIR length in NIR (blue) and SWIR (orange) for different input polarizations.
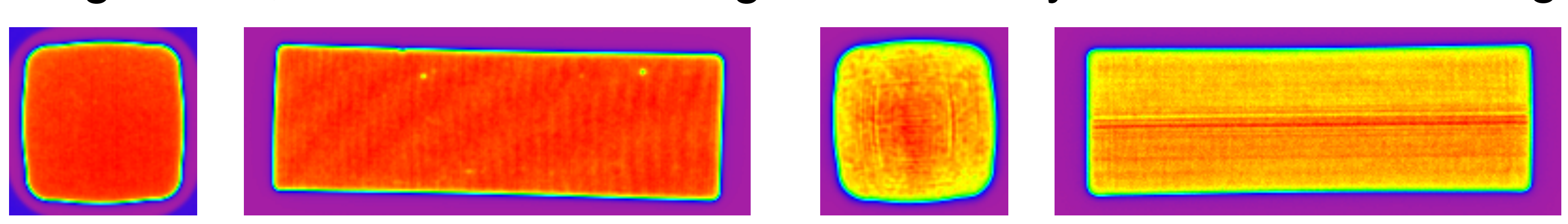

SWIR
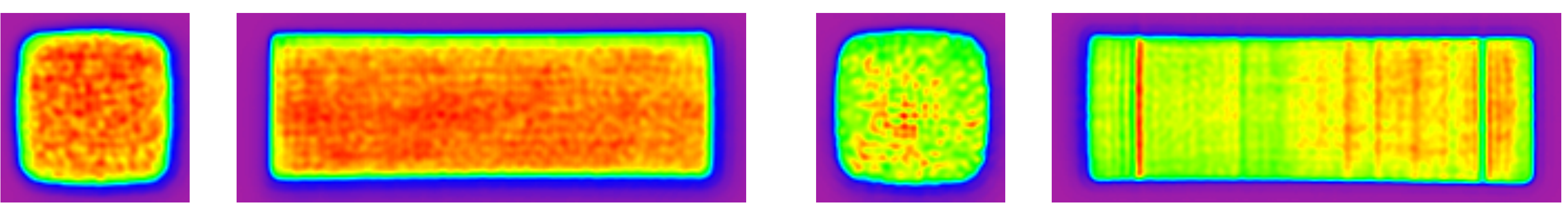

Degree of polarization:

$D=\frac{E_{\max }-E_{\min }}{E_{\max }+E_{\min }}$
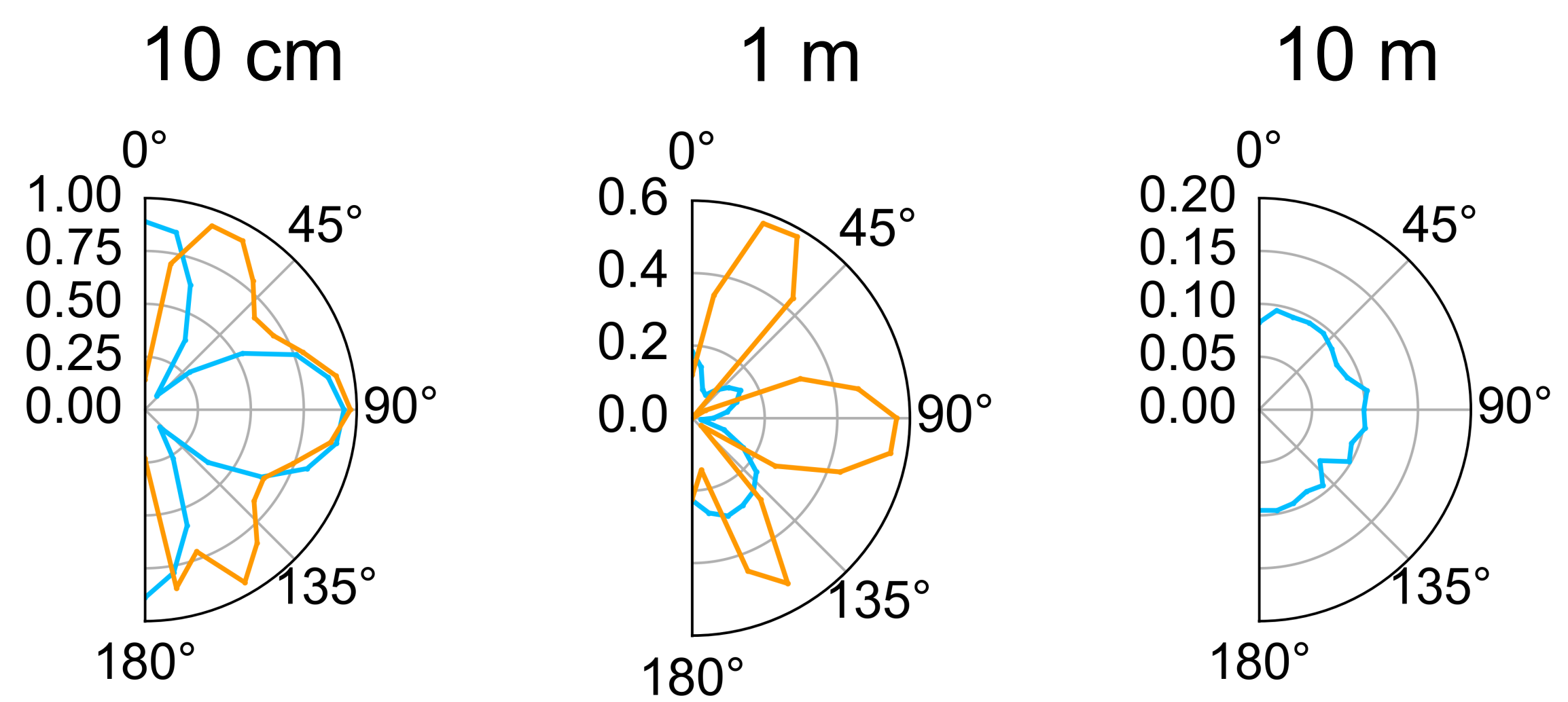

\section{Results}

Investigation of the broadening of the light cone caused by the fibers.

- Point illumination of the fiber input with different NAs,

- measurement of the far field,

- binning in $N A_{\text {Out }}$ ranges,

- cumulate and intersect (e.g. at $90 \%$ of the total energy),

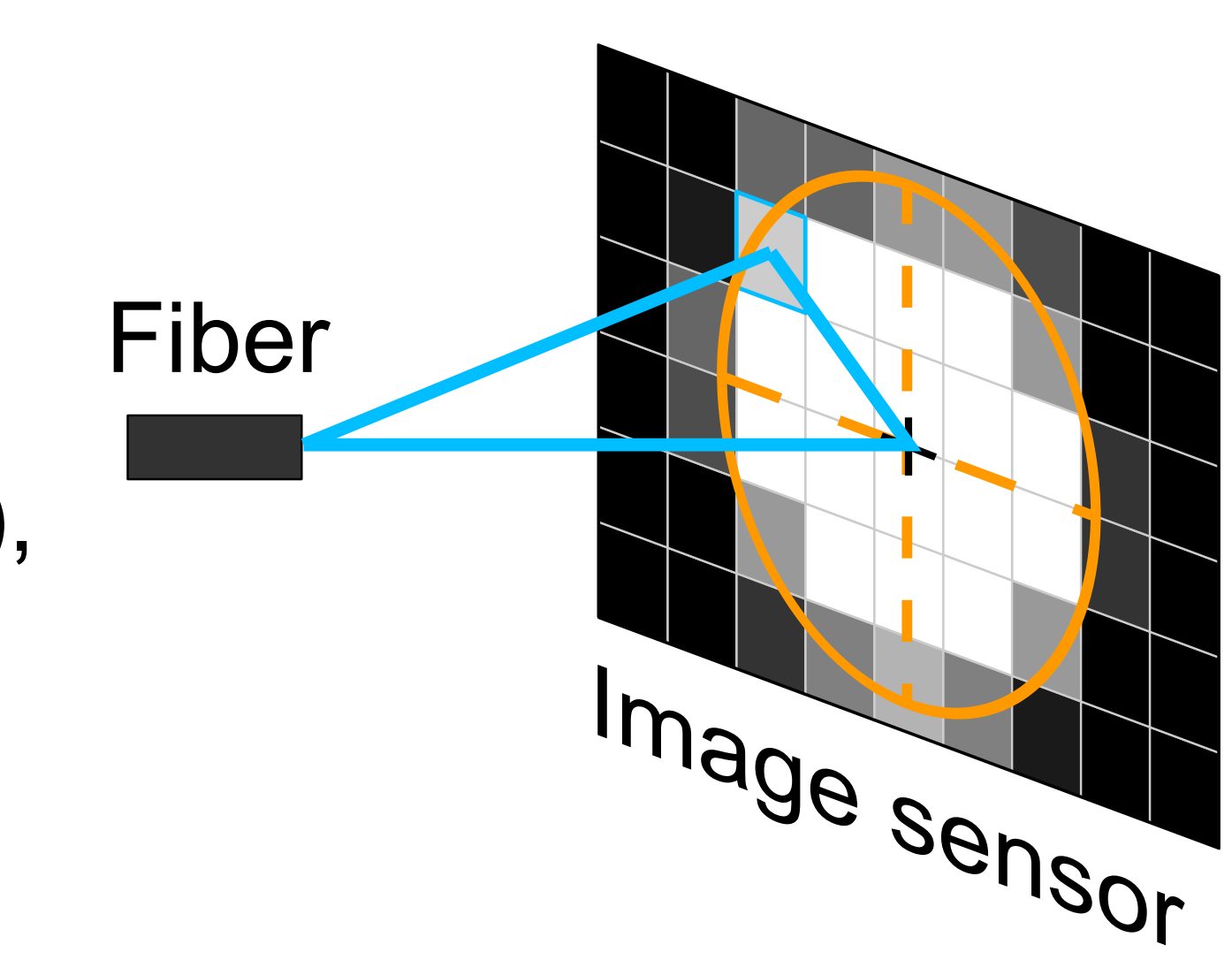

selected amount of energy.

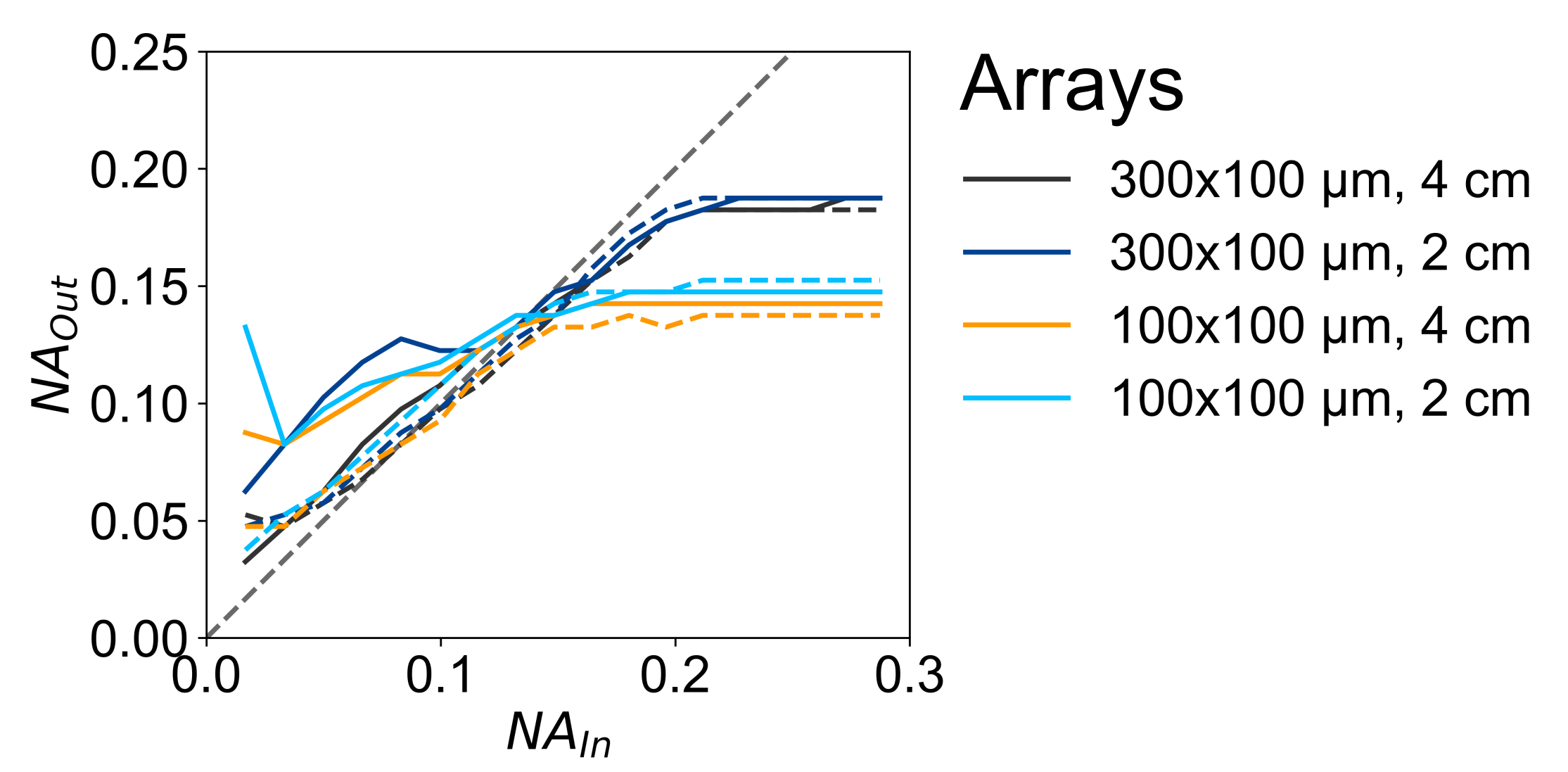

- General improvement of the light homogeneity for each device, fiber and wavelength.

- Some interference effects, dips, peaks and gradients left.

- More "noise" for SWIR than for NIR.

- Depolarization increases with fiber length.

- Unexpected increases of the FRD for the VIS wavelength (probably due to scattering)

- No influence of the fiber length on the FRD (Fiber output effect).

- The maximum guided NA is between 0.13 and 0.2 (flattening of the curve)

- Further improved homogeneity by vibrating the fiber. 